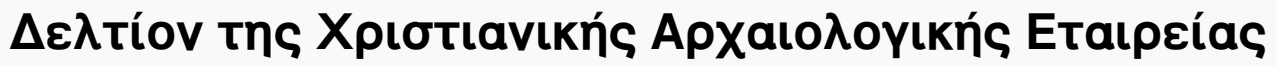

Tóp. 4 (1966)

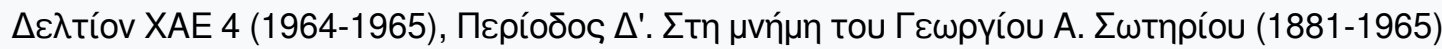

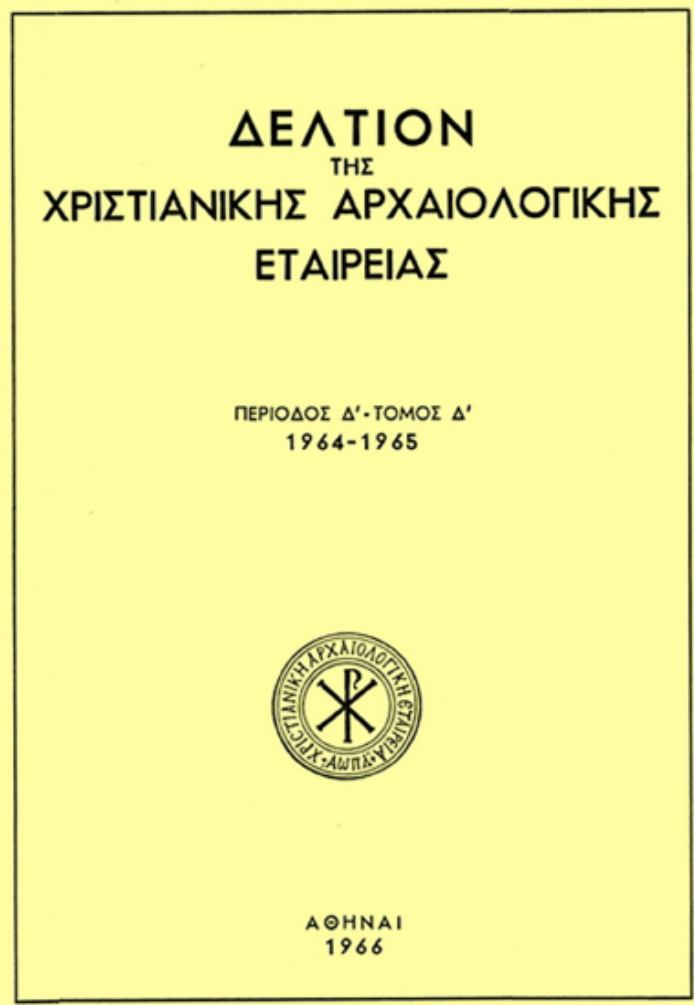

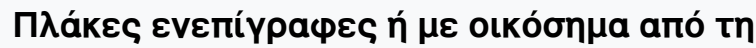

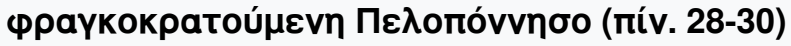

Antoine BON

doi: $\underline{10.12681 / \text { dchae.758 }}$

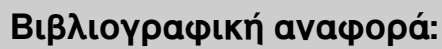

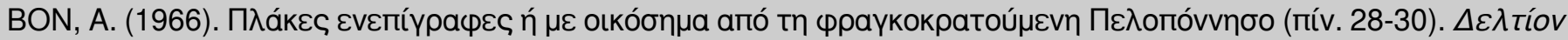

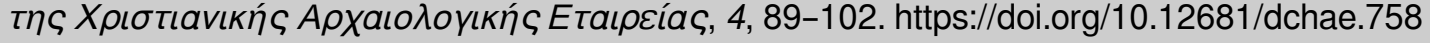




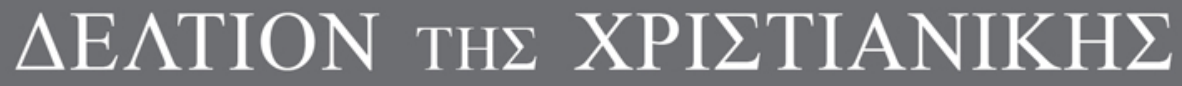 APXAIO $\Lambda$ OГIKH $\Sigma$ ETAIPEIA $\Sigma$}

Pierres inscrites ou armoriées de la franque Morée (pl. 28-30)

Antoine $\mathrm{BON}$

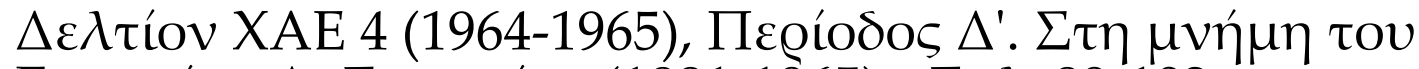

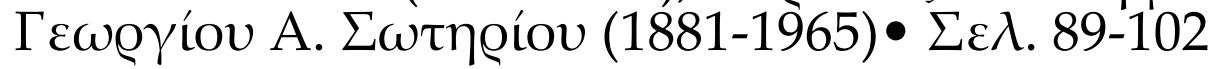

A@HNA 1966 


\section{PIERRES INSCRITES OU ARMORIÉES DE LA MORÉE FRANQUE}

(Pl. $28-30$ )

J'ai toujours été frappé par l'extrême rareté dans le Péloponèse de vestiges apportant un témoignage historique direct et précis sur la période de la principauté d'Achaie. Les chevaliers francs venus au temps de la $4^{\mathrm{e}}$ croisade, leurs descendants ou leurs successeurs ont élevé d'assez nombreux monuments, églises et forteresses, dont les ruines sont parfois considérables et dont certains ont conservé des fragments de sculpture intéressants pour l'histoire de l'architecture et de la décoration de style occidental en Grèce. Mais si l'on s'en tient aux pierres inscrites ou armoriées, il faut reconnaître qu'elles sont très peu nombreuses bien que la principauté ait duré plus de deux siècles. C'est pourquoi il n'est pas inutile de réunir ces documents, dont la plupart sont déjà publiés, mais de façon très dispersée.

A la différence des cités ou des régions où s'est étendue la domination vénitienne ou génoise, les territoires moréotes de la principauté n'ont conservé que très peu de blasons. Dans un mémoire publié en 1857, Rangabé a signalé sur le site de l'antique Pellène, en Achaïe, à quelque distance au Sud-Est de Xylokastro, une "grande plaque de tuf sur laquelle sont gravés deux écussons portant l'un une croix, l'autre un lion regardant à gauche "' ', dont nous reproduisons le dessin (fig. 1). La colline où était la ville antique est couronnée par les ruines d'un château médiéval pour lequel il n'est pas possible de proposer une identification sûre: peut-on reconnaître les armoiries? Le bloc est entouré d'un rebord formant cadre à l'intérieur duquel sont placés les deux écus; en haut les pièces du blason semblent déborder sur ce cadre; la partie inférieure manque. L'écu de gauche porte une croix, le chef présente trois gouttes ou pendants qui forment avec le cadre

1. R a n g a bé, Journal d'une excursion d'Athènes en Arcadie, dans Mémoires prés. par divers savants à l'Acad. des Inscr. et B. - Lettres, 1e Série V, 1e Partie, Paris 1857, pp. $326-329$, et pl. IV, fig. 15. 
de la pierre un lambel; celui de droite porte un lion rampant à la queue léopardée; la tête du lion déborde légèrement sur le cadre supérieur de la pierre et est surmontée, comme la croix, d'un arc de cercle dont la signification nous échappe. Croix et lion sont des pièces de blason fréquentes, donc difficilement identifiables.

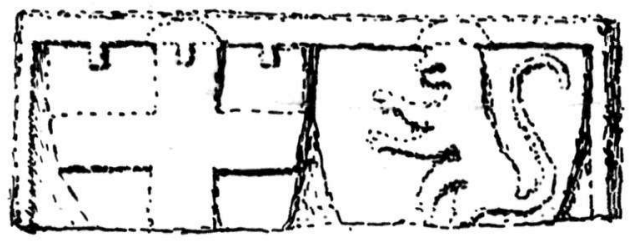

Fig. 1. Pierre trouvée à Pellène

On les retrouve sur un petit chapiteau déposé dans l'église Sainte-Sophie d'Andravida. C'est une pierre en forme de tronc de pyramide renversé, de section à peu près carrée $(0 \mathrm{~m}$. 314 sur 0,318 à la base, $0 \mathrm{~m}$. 375 sur 0,390 en haut), haute de $0 \mathrm{~m}$. 248. Chacun des côtés porte un écu placé entre une moulure supérieure faite d'une double baguette et une moulure inférieure en baguette simple, et encadré de deux fleurs en forme d'étoile à cinq pétales; sur les deux côtés légèrement plus petits, l'écu porte une croix ancrée, ou plutôt recercelée ; sur les deux autres côtés, l'écu est parti et porte en dextre (c'est-à-dire à notre gauche) un lion chargé d'une bande, en senestre une croix recercelée (fig. 2). Sans être d'un travail excellent, le dessin est assez habile : le lion a été en effet disposé de manière à donner une silhouette à peu près symétrique à la croix. Ces armoiries ainsi disposées sont en dextre celles du mari, en senestre celles de la dame: elles doivent être attribuées à Florent de Hainaut qui fut prince d'Achaĩe de 1289 à 1297 et à sa femme, la princesse Isabelle de Villehardouin. Le blason des Villehardouin, à la croix recercelée, figure d'une part en entier et seul, comme étant celui de la famille régnante, et d'autre part sur l'écu parti dont la moitié est réservée au blason de Florent de Hainaut, le lion d'or sur champ de gueules, chargé d'un bâton en bande, indiquant sans doute qu'il s'agit d'un cadet: Florent était le frère puiné du comte de Hainaut, Jean II d'Avesnes '.

1. Les armes de Florent sont représentées sur le sceau publié par G. $\mathrm{Schlumberger-Chal} \mathrm{and} \mathrm{on-Bl} \mathrm{anchet,} \mathrm{Sigillographie} \mathrm{de} \mathrm{l'Orient} \mathrm{latin,}$ Paris 1949, p. 185 n $^{\circ} 51$, pl. IX, 2. La houppe de la queue du lion, non visible sur le chapiteau d'Andravida, se retourne vers l'échine, mais ce n'est pas toujours 
Si l'on revient à la pierre de Pellène, on doit reconnaître que la croix simple surmontée d'une brisure sous la forme du lambel ne peut être celle des Villehardouin; ce pourrait être celle de Savoie; sur un sceau de Philippe de Savoie, prince d'Achaie de 1301 à 1307 comme second mari d'Isabelle de Villehardouin, on voit son écu à la croix
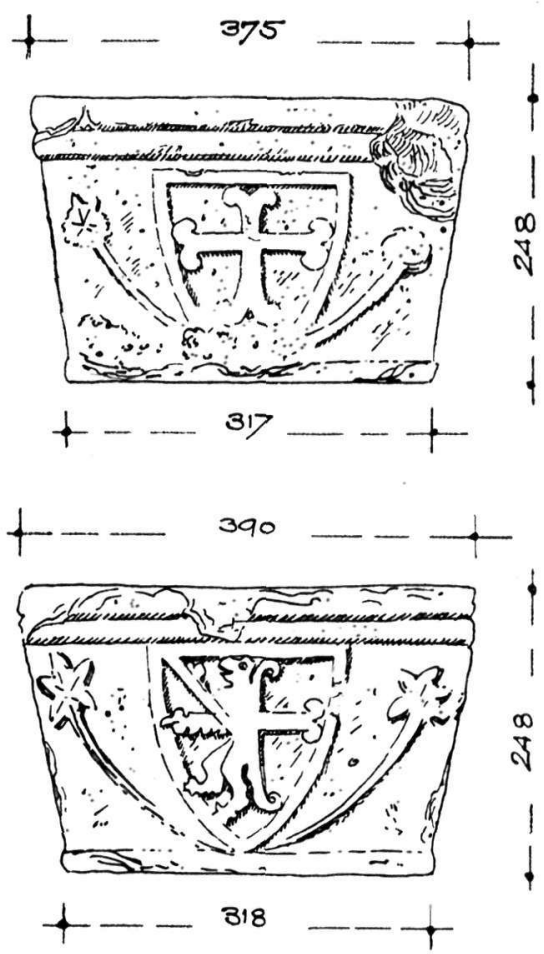

Fig. 2. Chapiteau à Andravida

chargé d'une bande, qui est également une brisure ${ }^{1}$. La croix de Savoie figure en même temps que le lion sur des documents, sceaux ou mon-

[e cas sur les armes de Hainaut où elle est souvent représentée retombant vers l'extérieur (queue “ léopardée »).

1. Voir le sceau publié par Bu chon, Atlas des nouvelles recherches, Paris 1845, pl. 25, 8, et par G. S chlu mberger, Sigillographie, pl. XX, 2. On retrouve ce blason placé au-dessus de celui de Villehardouin à la croix recercelée sur un sceau de la princesse Isabelle, $\mathrm{B} \mathrm{u} \mathrm{ch}$ o $\mathrm{n}$, Atlas, pl. $25,9,-\mathrm{S} \mathrm{chlu} \mathrm{m} \mathrm{ber-}$ g e r, Sigillographie, pl. XX, 3. 
naies, de représentants de la maison de Savoie qui gardèrent le titre de prince d'Achaie jusqu'au début du XVe siècle et tentèrent parfois de faire valoir leurs droits, mais je ne connais pas d'autre monument en Morée où croix et lion soient réunis ${ }^{1}$.

D'autres blasons se trouvent à Géraki, en Laconie. Les Francs ont occupé cette région vers la fin du premier quart du XIII e siècle; une baronnie fut créée, attribuée à un seigneur du nom de Guy de Nivelet, à qui succéda Jean. Géraki est le centre de cette baronnie : sur le site antique de Geronthrai s'était développée une agglomération importante jusqu'au XIIe siècle à en juger par le nombre des églises ${ }^{2}$; mais au XIII ${ }^{\mathrm{e}}$ siècle, le baron fit construire son château à quelque distance au Sud-Est à la pointe Nord d'une arête rocheuse qui convenait bien pour une forteresse, mais qui semble ne pas avoir été habitée jusque-là. Par la suite, la population vint s'établir sur la pente à l'Ouest du château, la moins raide, tandis que quelques constructions notamment des chapelles s'élevaient sur l'arête rocheuse longue de huit à neuf cents mètres ${ }^{3}$; elle a abandonné aujourd'hui ce site pour revenir à celui de la ville antique et byzantine, plus commode et pourvu d'une belle source.

Les pierres sculptées qui nous intéressent se trouvent dans l'église Saint-Georges située dans le château: c'est une église à trois nefs précédées d'un narthex couvert de trois calottes, mais la nef centrale et la nef septentrionale sont plus anciennes, tandis que la nef Sud et le narthex ont été construits plus tard ${ }^{4}$. La porte qui mène du narthex dans la nef centrale, et qui devait être primitivement une porte

1. B u ch on, Atlas, pl. XXIV, $12-13$, publie deux sceaux de princes titulaires d'Achaïe-Savoie, où l'on voit un lion rampant placé au-dessus d'un petit blason à la croix de Savois chargé d'une bande. Cf. la monnaie reproduite dans l' Enciclopedia italiana, I, 1929, p. 170. Mais il est peu probable que ce soit un de ces princes titulaires qui ait fait sculpter la pierre de Pellène.

2. L'intérêt des monuments byzantins a été signalé en particulier par G. S otiriou, E.E.B.E., I, 1924, p. 346, - et par Jacqueline Laf on $\mathrm{t}$ a in e, Le village “byzantin » de Ieraki, Reflets du monde, no 9, mai 1956, pp. 45 - 58.

3. Sur le site et sur l'ensemble des ruines de Géraki, voir Ramsay Traquair, Laconia. Mediaeval Fortresses, Ann. of the Brit. Sch. at Athens, XII, 1905/06, pp. $263-269$, à compléter pour les sculptures par A. J. B. W a c e, Laconia. Frankish Sculptures at Parori and Geraki, A. B. S. A., XI, 1904/05, pp. $138-145$, et pour les armoiries par A. V an de P u t, Note on the armorial Insignia in the Church of St. George, Geraki, A. B. S. A., XIII, 1906/07, pp, $281-283$.

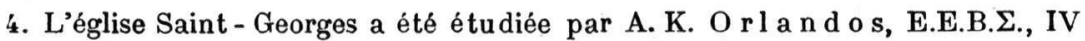
1927, pp. $348-349,351$; sur la date de la partie ancienne, v. infra. 
extérieure, est surmontée d'un arc brisé fait de quatre claveaux de pierre et d'une clé qui porte sculpté un blason échiqueté (fig. $3 \mathrm{a}$ ). A l'intérieur se trouve placé contre le mur septentrional de la nef Nord près de l'iconostase, un proskynitari, qui se présente comme un édicule dont la façade en marbre local, haute d'environ $1 \mathrm{~m} .65$ (sur
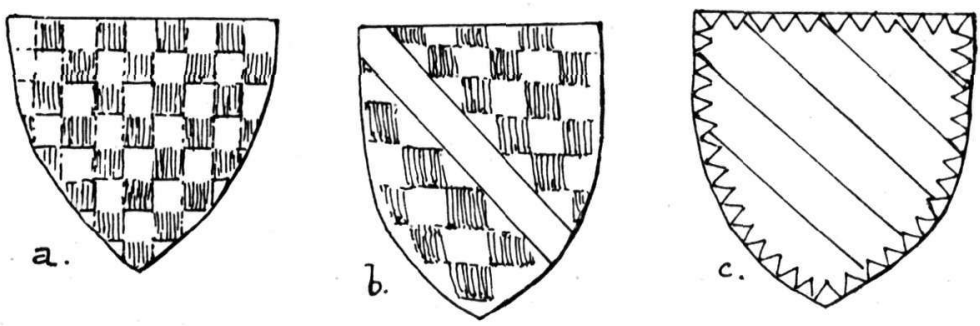

Fig. 3. Blasons de Geraki et de Patras

un soubassement maçonné de $0 \mathrm{~m} .35$ ) et large de $2 \mathrm{~m} .10$, est richement décorée de sculptures à jour et se termine par un gable assez aigu (Pl. $28 \mathrm{a}$, cf. le dessin très précis de R. Tr a qu a ir, A.B.S.A, XII, 1905/06, pl. IV). Au sommet est représenté un écu losangé ou échiqueté chargé d'une bande; il est surmonté d'un motif indistinct qui ne semble pas avoir été une couronne ni un panache (fig. $3 \mathrm{~b}$ ). De plus, de part et d'autre de l'arcade sous laquelle s'ouvre la niche, sont sculptés en faible relief, à gauche, un croissant entre six étoiles, deux en haut, une dans la concavité du croissant, trois alignées en bas, à droite, une fleur de lys cantonnée de quatre petites roses; chacun de ces groupes de motifs pourrait facilement constituer un blason. Enfin, dans un autre monument, une chapelle située à l'extrémité Sud de l'arête rocheuse, se trouve un fragment de peinture murale qui représente un combat dans lequel Josué attaque une cité des Amorrhéens: les personnages ont des costumes médiévaux qui semblent bien être ceux des chevaliers occidentaux du XIII' siècle; Josué à cheval porte un bouclier dont le champ est orné d'un croissant cantonné de quatre étoiles ${ }^{1}$.

Les deux écus placés en haut de la porte de la nef centrale de Saint-Georges et sur le gable du proskynitari portent certainement de vrais blasons; ils n'ont pu être exécutés que sur l'ordre de seigneurs

1. C'est l'église no 4 du plan de R. Traquair, A.B.S.A., XII, 1905/06, p. 265 , plan, fig. 2 p. 262 , fresque, pl. II, 1. Cf. J. L o n g n o n, L'empire latin de Constantinople, Paris 1949, pp. 215 - 216. Sur la fleur de lys, voir infra. 
francs : on peut en conclure que la partie ancienne de l'église date de l'époque où ceux-ci étaient présents ', de même que le proskynitari, même si celui-ci est légèrement postérieur au bâtiment même. Mais si cette indication est précieuse, on voudrait savoir quelles sont ces armoiries. Tout d'abord s'agit-il du blason de la même famille? C'est ce que croit pouvoir admettre A. Van de Put: pour lui c'est un blason losangé qui, pour une branche ou un membre de la famille est chargé d'un bande, qui n'est qu'une simple brisure. Cependant le blason de la porte est nettement échiqueté et non losangé, comme l'a vu R. Traquair (A.B.S.A., XII, pp. 265 -266); si les deux blasons appartiennent à la même famille, ce que l'on est tenté d'admettre, je pense que c'est plutôt celui du proskynitari qui est légèrement déformé, le sculpteur ayant dessiné, en suivant les lignes de la bande, les points de l'échiquier légèrement en diagonale et non en carrés placés verticalement; le blason original est vraisemblablement échiqueté à neuf points. A qui appartient-il? Le plus vraisemblable est que ce soit celui des barons du lieu; mais la famille de ces barons est bien mal connue. On transcrit traditionnellement le nom de Nivelet en Nivelles, lieu qui se trouve dans le Nord de la France, près de Valenciennes. A. Van de Put a supposé que Nivelet était une déformation du nom des Neuville (nom très répandu en France!): plusieurs personnages de ce nom sont venus en Orient au XIe, au XII e et au XIII e siècle; au XIVe, des Nevile, Neviles ou de Nivilis sont à Chypre, et sur deux tombeaux à Nicosie dont l'un porte le nom "Johan d' Nevilles ", figure le blason losangé, ou losangé chargé d'un lambel à trois pendants ${ }^{2}$. Le rapprochement est intéressant, mais est-il sûr? N'est-ce pas ce rapprochement même qui a poussé $\mathrm{A}$. Van de Put à considérer les blasons de Géraki comme losangés, ce qui est inexact pour l'un et douteux pour l'autre? De plus l'équivalence Neuville-Nevile-Nivelet ne s'impose pas: le nom de Nivelet peut tout aussi bien être une forme authentique et désigner un seigneur venu d'un hameau de ce nom situé dans le département du Jura, région qui a fourni à la Quatrième croisade les seigneurs de Rosières et de Dramelay qui devinrent respectivement barons d'Akova

1. Je ne vois aucune raison de dater les deux nefs anciennes de SaintGeorges du XIIe siècle: ce site ne devait pas encore être habité alors et rien dans la construction n'oblige à préférer le XIIe ou XIIIe siècle; les blasons ne peuvent être antérieurs à 1225-1230 et aucun indice ne permet de supposer qu'ils aient été placés après-coup dans un monument existant déjà.

2. Van de Put, 1.1., p. 282. 
et de Chalandritsa '. Mais nous ignorons quelles étaient les armes des seigneurs de Nivelet en France.

Quant au bouclier de Josué, les armoiries qu'il porte ne peuvent être qu'un blason de fantaisie comme l'a bien vu A. Van de Put; et il y a tout lieu de supposer que les motifs placés de part et d'autre de l'arcade sur le proskynitari n'ont qu'un rôle décoratif. En résumé si ces blasons ne révèlent pas tout ce qu'on voudrait savoir, ils apportent un renseignement précieux sur la date des monuments, en particulier pour le curieux proskynitari : exécuté dans le second tiers du XIIIe siècle, il est certainement l'œuvre d'artistes grecs, car si l'influence occidentale est révélée par le blason, par la forme de l'arcade tréflée de proportion assez maladroite, il est évident que le travail “à jour » du marbre ni les motifs décoratifs ne sont occidentaux. C'est un exemple du travail soigné qui pouvait être exécuté à cette époque, et dont la pierre tombale de la princesse Agnès est l'équivalent ${ }^{2}$.

Cette dalle funéraire est de beaucoup la plus importante de trois pierres qui offrent simultanément inscription, armoiries ou decor, les deux autres étant celles d'un seigneur enterré au couvent des Vlachernes

1. Cette hypothèse m'a été suggérée par M. Paul Deschamps, que je prio de bien vouloir accepter ici mes remerciements.

2. L'opinion d'A. J. B. W a c e, 1.1., p. 145, selon laquelle il faudrait voir ici un travail "sarrazin », ne me paraît nullement justifiée. R. T r a q u a i r, A.B.S.A., XII, 1905/06, pp. 267-269, signale au contraire la ressemblance avec des monuments de l'Italie méridionale, mais en considérant à tort que ceux-ci doivent être attribués au XIVe siècle; d'après les renseignements que lui fournit ultérieurement W. Miller, deux des monuments : une chaire dans le dôme de Bitonto, et un des tombeaux des Falcone dans l'église Sainte-Marguerite de Bisceglie en Pouille sont datés, respectivement de 1229 et de 1246, A.B.S.A., XIII, 1906/07, pp. $283-284$; ces dates correspondent bien avec l'époque où a pu être construit Saint-Georges et où ont pu être sculptés les blasons; il est invraisemblable que ce soient les armes de feudataires des rois Angevins, de Naples, au XIVe siècle, comme le dit aussi R. Traquair, car la région est alors grecque depuis longtemps.- Au Sud de Monemvasie, près du village de Geroumana, l'église de la Pantanassa garde des traces de style occidental, bien que de structure purement byzantine, puisqu'elle est construite sur plan en croix grecque inscrite; la porte de la façade Ouest a une arcade tréflée, et celle qui mène du narthex à la nef centrale présente le même arc brisé que celle de Saint-Georges, la clé en porte également un blason, mais il est effacé. Pour la date de l'église, le XIIIe siècle, que donne G. S o-

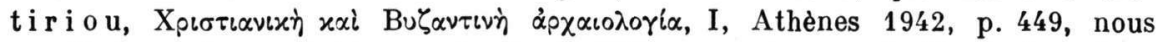
paraît donc plus probable que la fin du XIIe proposée par A. K. Orlandos, A.B M.E., I, 1932, pp. $139-151$. 
d'Elide et d'un archevêque de Patras. Je ne ferai qu'en rappeler ici les principaux caractères et l'intérêt ${ }^{2}$. C'est la partie supérieure d'une plaque de marbre large de $0 \mathrm{~m} .82$, conservée sur une hauteur de $0 \mathrm{~m} .68$, ce qui représente environ le tiers de sa longueur totale, trouvée à Andravida sur l'emplacement de l'église Saint-Jacques, aujourd'hui disparue, où avaient été enterrés les princes Geoffroy Ier, Geoffroy II et Guillaume de Villehardouin. Le fragment est couvert d'un décor sculpté entouré d'une inscription dont le début et la fin sont préservés et donnent le nom du personnage: Agnès, fille du despote Michel (et troisième femme du prince Guillaume II), et la date de sa mort, le 4 janvier 1286 (Pl. 29). L'intérêt de cette pierre, qui porte la seule inscription en français que je connaisse dans le Péloponèse, est multiple : au point de vue historique, elle apprend la date exacte de la mort de la princesse et le lieu de son inhumation, ignorés jusqu'ici. Au point de vue artistique, elle constitue un document exactement daté qui frappe, car à côté de l'inscription en français, le décor est d'inspiration purement byzantine avec la croix en passementerie, les quatre paons d'un dessin lourd mais parfaitement reconnaissables, et au revers de la pierre, la rosace timbrée d'une croix, le poisson, l'oiseau, l'arbre esquissés d'une simple incision. Un détail pose cependant un problème, ce sont les quatre petits reptiles placés au centre du motif, leur aspect tacheté me les a fait considérer comme des salamandres; mais je n'ai encore rencontré aucun autre exemple de ces animaux ni dans l'art byzantin ni dans l'art occidental de cette époque; leur association avec les paons s'explique par une intention symbolique: l'oiseau représente l'éternité triomphant du reptile représentant le monde infernal. L'examen attentif et répété de la pierre m'a convaincu que tout le travail a été exécuté dans le même temps, c'est donc l'œuvre d'un artiste de la fin du XIII' siècle. gardant sous la domination franque, il est vrai pour une princesse d'origine grecque, des motifs byzantins. La pierre ne porte aucune trace d'usure.

Plus récente de trois quarts de siècle est la dalle funéraire qui se trouve au couvent des Vlachernes à quelques kilomètres d'Andravida et du port médiéval de Klarentsa; la partie supérieure seule conservée, large de $0 \mathrm{~m} .725$ sur une hauteur à peu près égale, garde l'épitaphe

2. J'ai donné une description de cette pierre: Dalle funéraire d'une princesse de Morée (XIIIe siècle), dans Monuments et Mémoires (Fondation Piot), XLIX, 1957, pp. $129-139$. 


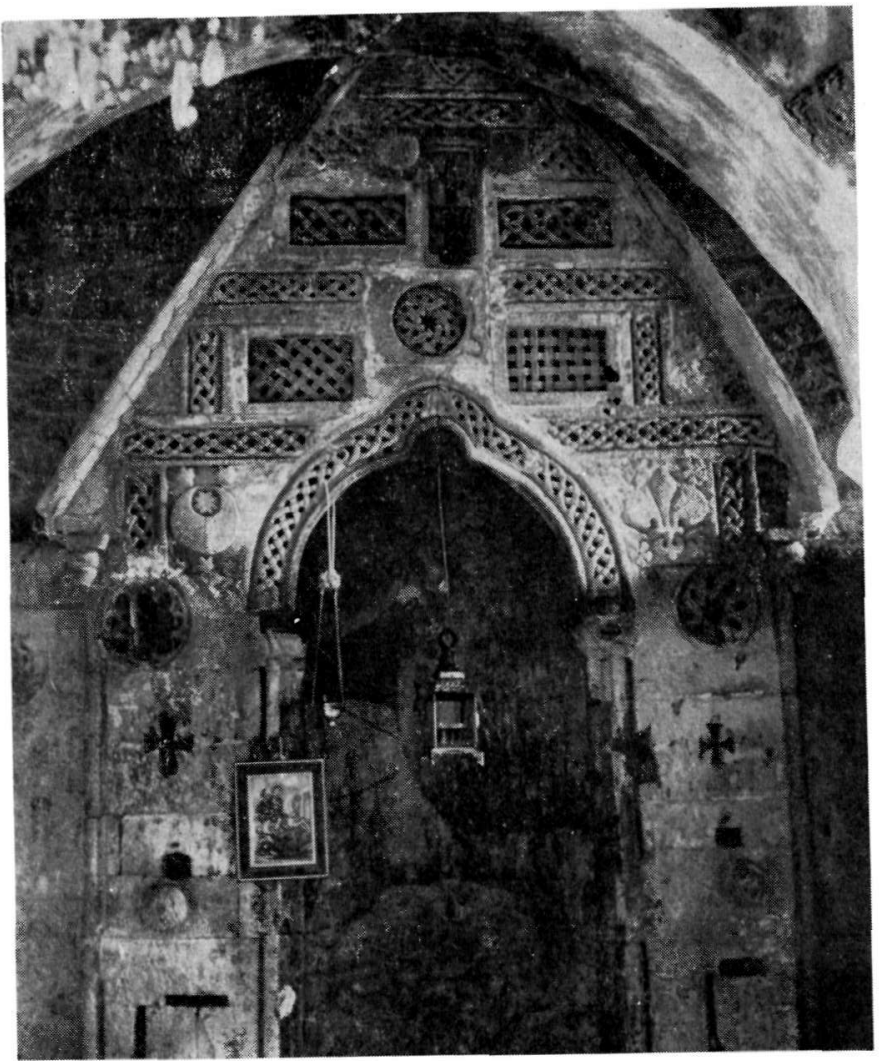

a. Proskynitari à St. Georges, Geraki

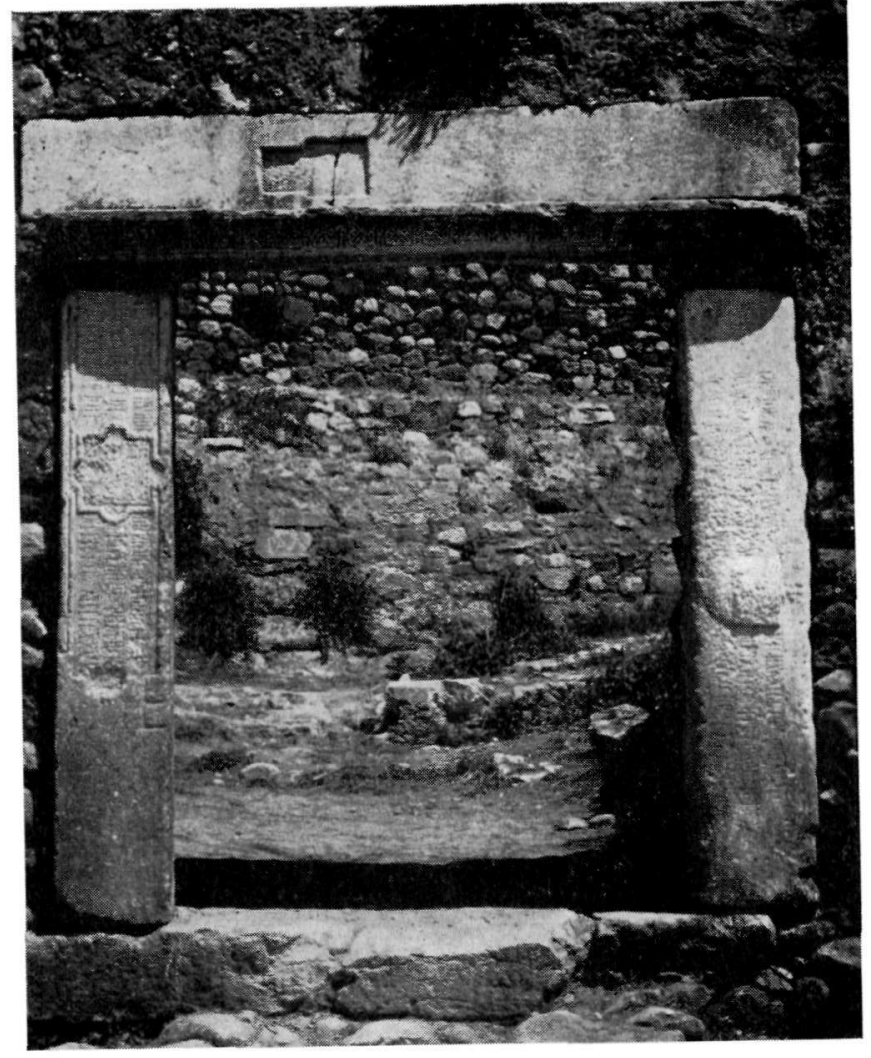

b. Ancienne porte au Chateau de Patras 

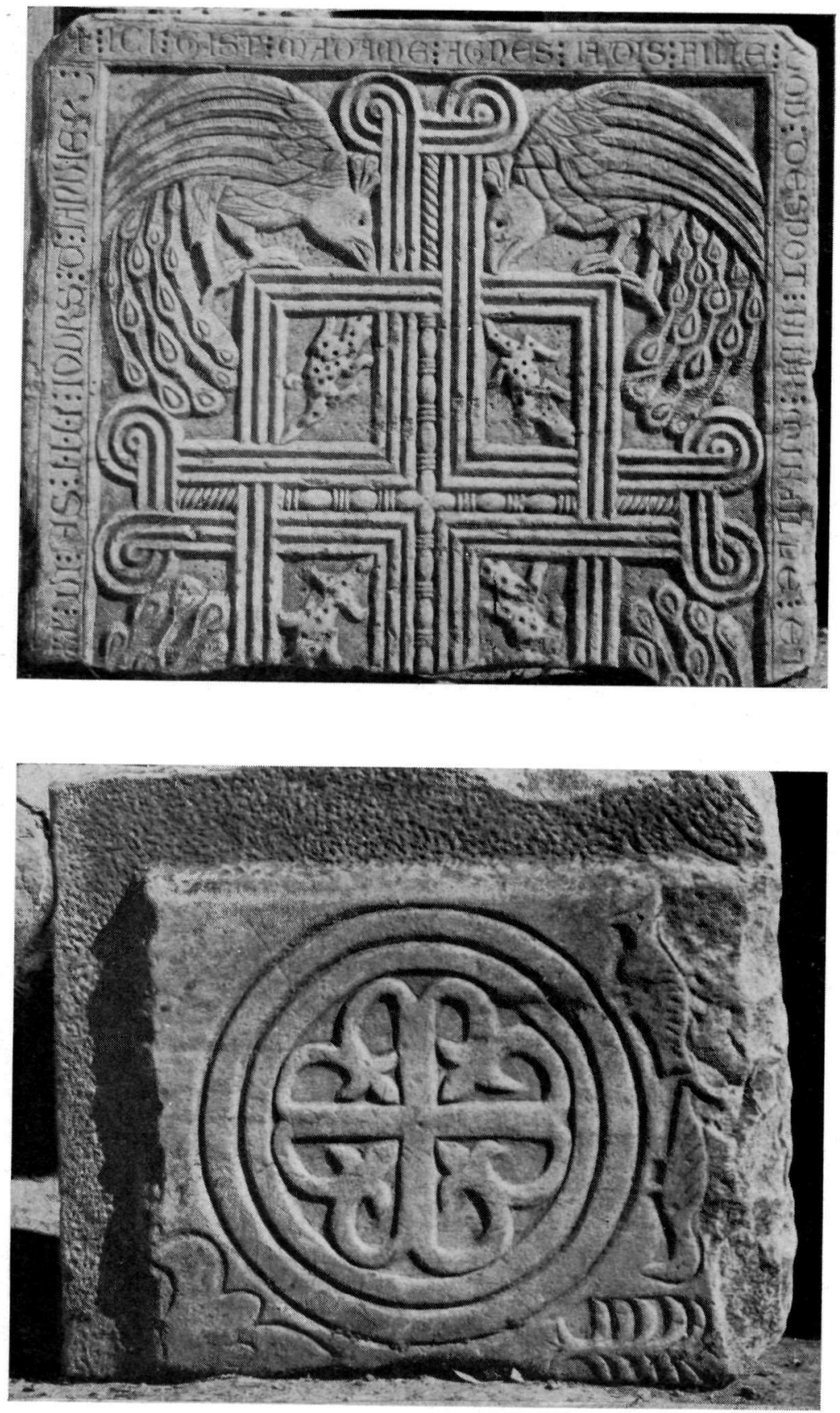

Face et revers de la pierre tombale de la princesse Agnès à Andravida 

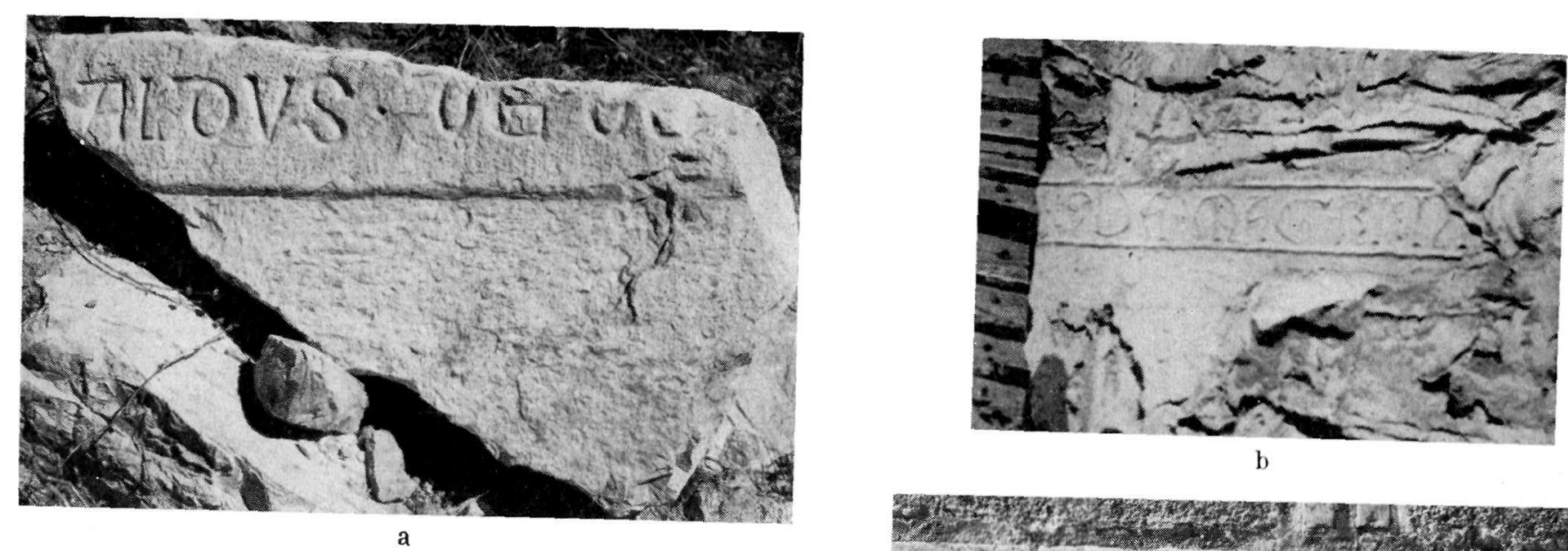

b

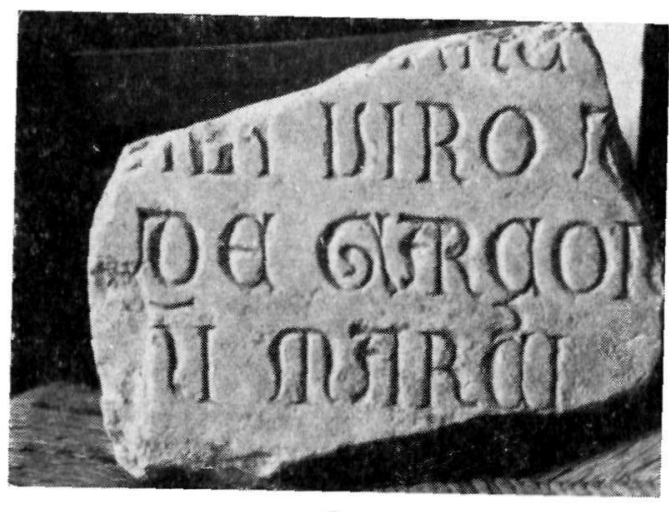

Inscriptions a. de Chlémoutsi, b. de Klarentsa,

c. de Cornon (musie de Kalamata), d. graffiti aux Vlachernes

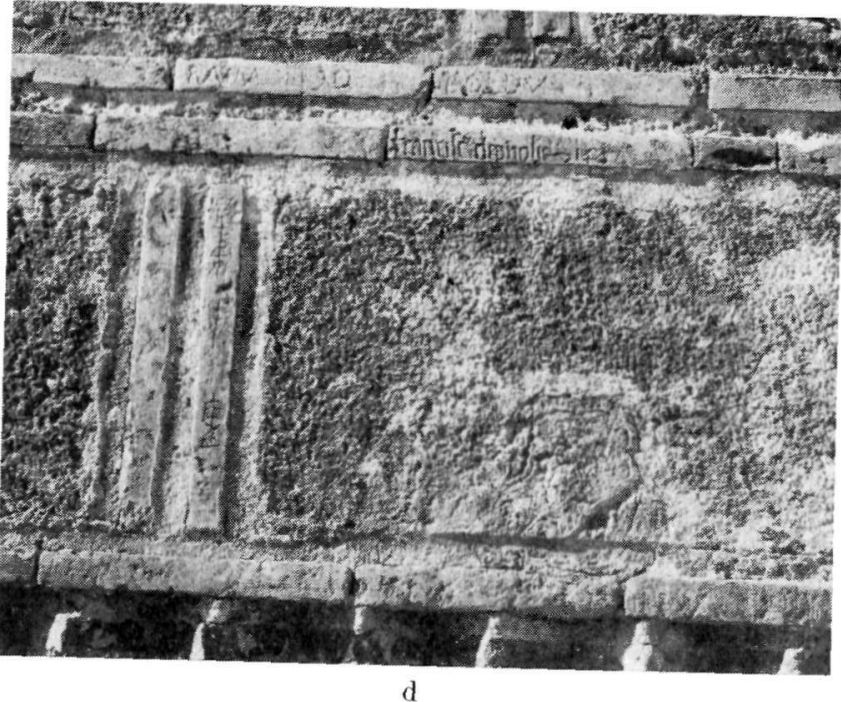


et un écu chargé d'un lion; le logement de l'anneau qui permettait de la soulever se voit encore, mais elle été enlevée sans doute depuis longtemps du tombeau qu'elle devait couvrir (fig. 4). L'inscription en latin est gravée avec soin en lettres hautes de $2 \mathrm{~cm} .5 \mathrm{à} 3 \mathrm{~cm}$.; malheureusement le calcaire gris de la dalle s'écaille par endroits, pla fin des

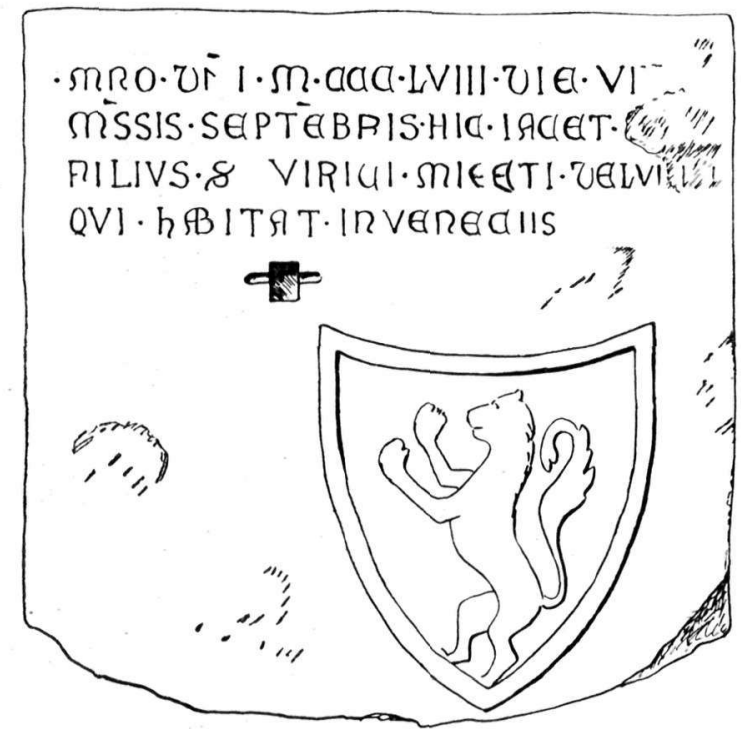

Fig. 4. Pierre tombale au couvent des Vlachernes

lignes à droite notamment est à peu près illisible. Buchon a vu la pierre et en donne la lecture suivante ${ }^{1}$ :
ANNO DNI. M. CCG. LVIII. DIE XX
MENSIS SEPTEMBRIS. HIC JAGET SEME-
NILIUS Sti VIRIDI-MILETI DE LUCINIA
QUI HABITAT IN VENECIIS.

L'ensemble est clair; les mots sont séparés par des points, les lettres omises sont indiquées par un trait horizontal au-dessus de la

1. Bu chon, Grèce continentale et Morée, Paris 1843, p. 513, et Atlas, pl. 40, no 2. La pierre tombale a été depuis lors signalée par S p. Lambros, N. 'E $\lambda$., XIII, 1916, p. 481, qui en donne une lecture très inexacte, puis par R. T raqu a ir, Journ. of the Royal Inst. of Brit. Arch., 3e Serie, XXXI, 1923, p. 80, qui reproduit, avec des erreurs le texte de Buchon; A. K. Orlandos, dans son important article sur les Vlachernes d'Elide, 'A $\rho$. 'E $\varphi ., 1923$, p. 12, a publié à son tour une lecture qui ne me parait pas définitive. 
ligne. A la ligne 1, seule la fin est douteuse, il s'agit du jour du mois, pour lequel les signes visibles actuellement sur la pierre semblent donner plutôt VII ou VIII, mais cela a peu d'importance; la fin de la ligne 2 est aujourd'hui illisible, mais le nom lu par Buchon, Semenilius est impossible; en fait la première lettre de la ligne 3 est un $\mathrm{F}$ et non un $\mathrm{N}$, on lit donc FILIUS normalement suivi d'un génitif que nous proposons plutôt de restituer $\mathrm{S}$ (erenissimi) VIRI ${ }^{1}$; le nom du personnage, à la fin de la ligne 2, devait être très bref. La suite est beaucoup plus obscure ; le Sti Viridi-mileti de Buchon est difficilement acceptable, il faudrait traduire "(fils de) Saint-Vert seigneur de Lucinia "; nous transcririons plus volontiers (filius S(erenis simi) Viri...); il n'y a pas, il est vrai, de point entre siri et $d i$; mais les lettres mileti de Buchon restent aussi énigmatiques, car on attendrait ici miles ou militis.

Reste la fin de la ligne 3, que Buchon a lu DE LUCINIA. Bien que l'espace sur la pierre soit étroit, il est possible que le graveur ait en effet serré les lettres pour éviter de couper le nom, mais ce qu'on voit aujourd'hui sur la pierre est bien difficile à interpréter. Le blason peut-il apporter quelque lumière? Le lion ${ }^{2}$ est une pièce très fréquente ; ce n'est pas celui de Hainaut; mais il est proche de celui des Lusignan qu'on voit sur les monnaies de Chypre seul ou associé à la croix de Jérusalem $^{3}$. Vers le milieu du XIVe siècle, les rapports sont fréquents entre les Lusignan et la Morée. Une pierre sculptée rapelle la présence d'une dame de cette famille dans le Péloponèse, c'est le monogramme d'Isabelle de Lusignan devenue despine de Mistra, auprès duquel sont gravées ses armes avec le lion et deux croix de Jérusalem ${ }^{4}$. D'autre

1. Il est de règle de donner le nom du père du personnage; ainsi l'épitaphe de Louis, fils du roi Robert et de Yolande d'Aragon, né en 1301, mort en 1310 et enterré en l'église S. Lorenzo Maggiore à Naples porte à la ligne 2: Filius Serenissimi Principis Roberti..., C. Minieri-Riccio, Archivo storico per le Provincie napoletane, VIII 1883, p. 595.

2. Le dessin de Buchon représente le blason chargé d'une fasce dont je n'ai vu aucune trace sur la pierre.

3. Voir G. Schlu mberger, Numismatique de l'Orient latin, pl. VI, no $15-19,23,26-28,-$ VII, no $2-6,9-10,12-13,16,18,24-25,-$ VIII, no $1-3$.

4. Cf. G. Mille t, Monuments byzantins de Mistra, pl. LVIII, no 1, 3. Sur cette inscription, sur Isabelle de Lusignan et sur la famille en général, voir G. Millet, Inscriptions inédites de Mistra, B.C.H., XXX, 1906, p. 453,-D. A.

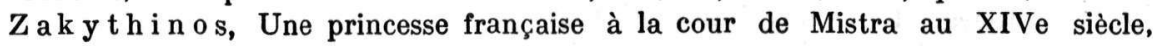
R.E.G., XLIX, 1936, pp. 62 - 76, et en dernier lieu S t. B i n o n, Guy d'Arménie et Guy de Chypre, Isabelle de Lusignan à la cour de Mistra, A.I.P.H.O.S , V, 1937 (Mélanges E. Boisacq, I), pp. 124-142. 
part la princesse d'Achaïe Marie de Bourbon avait été mariée d'abord avec Guy de Lusignan et avait eu de ce premier mariage un fils, Hubert de Lusignan, qui devait tenter plus tard d'occuper la principauté. Si la dalle du couvent des Vlachernes portait réellement DE LUCINIA, faut-il supposer qu'il s'agit d'une forme latine inhabituelle du nom des Lusignan? la tombe serait-elle celle d'un membre inconnu de cette famille? On lirait ainsi : «L'an du Seigneur 1358, le ... du mois de septembre. Ci gist .. . fils du S(érénissime?) Seigneur de ... . . . de Lusignan, qui habite (= habita) à Venise "). De toute façon, le personnage qui fut enterré aux Vlachernes devait être important et avait dû mourir, résidant à Venise, alors qu'il se trouvait à Klarentsa en escale ou pour affaires.

A Patras enfin, la porte d'une barbacane placée devant l'entrée du réduit dans le château qui domine la ville était naguère encore encadrée de grands marbres sans doute antiques, qui avaient déjà été remployés au Moyen Age avant d'être maçonnés dans cette porte à l'époque turque ou vénitienne ( $\mathrm{Pl} .28 \mathrm{~b})^{1}$. Celui qui formait le seuil ne présentait aucun détail notable; le linteau était constitué de deux blocs superposés, l'un relativement mince dont la tranche antérieure taillée en cavet droit était ornée d'un fin relief byzantin de rosaces placées dans un entrelac, l'autre au-dessus plus épais portait un encastrement, preuve du remploi; enfin les deux marbres dressés en forme de parastades à droite et à gauche de la porte présentent chacune un blason placé au centre d'un inscription; les blasons sont bandés avec une bordure dentelée (fig. $3 \mathrm{c}$ ); un des blocs long de $1 \mathrm{~m} .20$ sur $0 \mathrm{~m}$. 30 environ porte le texte en latin, gravé en caractères d'un gothique italien fleuri :

\begin{tabular}{|c|c|}
\hline MONUMENTU(M) ET ARMA & DOMINI PANDOLP[HI M] \\
\hline ALATEST[AE] ARCHI & EPISCOPI PATRA $[\ldots]$ \\
\hline AEDIFICATORI[S] HUJ(US) & ECGLESIE DCCGCXXVI \\
\hline
\end{tabular}

L'autre bloc, de $1 \mathrm{~m} .44$ de longueur sur $0 \mathrm{~m}$. 285, donne le texte grec :

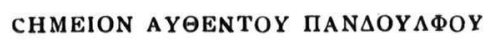

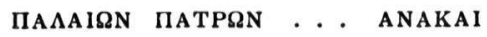
[EK] T(OI)C MALATECTOIC M(HT)POПOAITOY

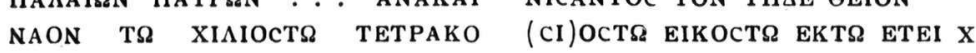

1. Ces blocs sont signalés et dessinés dans A. B 1 o u e t, Expédition scientifique de Morée. Architecture, sculptures, inscriptions..., III, Paris 1838, 64 no 7 et 8 , pl. 85. L'inscription grecque est reproduite dans $\mathrm{B} \mathrm{oe} \mathrm{ckh}$, C.I.G., IV, no 8776 , et par Thomopoulos, $\Delta$. I.E.E., I, p. 523 . 
Je n'insisterai pas sur ces documents : le texte, malgré quelques lettres effacées, est suffisament clair et ne pose pas d'autre problème que l'identification des travaux éxécutés dans une église que l'on peut supposer être la grande église de Patras, consacré à Saint-André. Le personnage de Pandolfo Malatesta est connu : c'est le dernier archevêque latin de Patras : intronisé en 1424, il est absent de la ville quand celle-ci revient aux Grecs en 1430, et meurt en Italie en $1441^{1}$. C'est précisément l'époque où la principauté elle-même achève de disparaitre obscurément. Si nous rappelons au passage cette inscription bien qu'elle soit à l'extrême limite du domaine qui nous intéresse, c'est qu'elle a aujourd'hui disparu ${ }^{2}$.

A ces documents importants on ne peut ajouter que quelques fragments d'inscriptions en latin dont la gravure indique nettement l'époque par sa ressemblance avec les épitaphes de la princesse Agnès ou du tombeau des Vlachernes. A Kyllini, près du site de Klarentsa, dans le mur d'une petite maison construite en 1960 a été encastré un

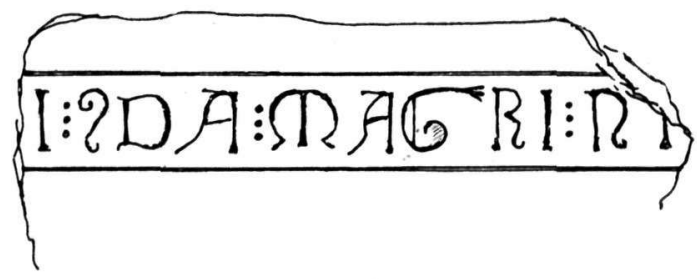

Fig. 5. Inscription à Klarentsa

fragment de marbre de $0 \mathrm{~m} .46$ de long sur une largeur maxima de $0 \mathrm{~m} .28^{3}$ : sur le bord supérieur, entre deux lignes incisées, court une inscription en lettres de 6 à $6 \mathrm{~cm}$. 5, où le seul mot lisible est celui de MAGISTRI (fig. 5 et Pl. $30 \mathrm{~b}$ ). Au-dessous du château de Chlémoutsi, dans le cimetière du village, des travaux récents à l'église Saint-Démètre ont fait sortir du sol un autre fragment de marbre, de $0 \mathrm{~m} .60$ de long

1. Cf. $\mathrm{H}$ o p f, Griechenland im Mittelalter, II, p. 82 et n. 55,-E. G e r l a n d, Neue Quellen zur Geschichte des lateinischen Erzbistums Patras, Leipzig 1903, pp. $64-69$.

2. Les marbres portant ces inscriptions ont été enlevés par les troupes italiennes d'occupation au cours de la dernière guerre mondiale.

3. Ce fragment m'a été signalé par M. Dinos Psychoyios que je remercie ici et dont je me plais à reconnaître la connaissance profonde de tout le passé de l'Elide et l'extrême obligeance. 
sur $0 \mathrm{~m} .37$ de large : c'est le bord d'une dalle funéraire, présentant un rebord sur lequel on lit en lettre de $5 \mathrm{~cm}$. 5 de haut:... ALDUS DE ... (Pl. 30 a). Enfin le musée de Kalamata conserve un fragment de marbre de $0 \mathrm{~m}$. 36 sur $0 \mathrm{~m}$. 28, provenant de Coron, donc vénitien mais datant probablement du XIII ${ }^{e}$ ou du XIVe siècle, d'après la gravure, et où l'on reconnaît le nom d'une famille vénitienne: $\mathrm{DE}$ GARÇON... (Pl. $30 \mathrm{c})$.

Je n'ai relevé nulle part de graffiti datant de l'époque franque, alors que ce genre de témoignages est fréquent pour les époques postérieures. Les plus anciens que j'aie vus se trouvent à l'église du couvent des Vlachernes d'Elide, gravés sur la tranche des briques, à l'abside ou sur le mur Sud ${ }^{1}$; de ce côté de l'église, à droite de la porte on lit sur une brique les noms RAIMUNDO BOLDUS, qui sont ceux d'un personnage inconnu : puis, au-dessous, sur une brique verticale, deux monogrammes surmontés d'une croix et dont l'un est accompagné de la date 1580; enfin, de nouveau sur une brique horizontale, gravé avec beaucoup de soin : (Francisco de Priolis 1447) (Pl. $30 \mathrm{~d}$ ); or on sait que Francesco de Priolis fut le premier gouverneur de Chypre en $1449^{2}$; si nous relevons de détail au passage, e'est qu'il montre que les voyageurs se rendant de Venise en Orient faisaient escale à Klarentsa et pouvaient aller visiter le couvent voisin des Vlachernes, bien que aucun texte ancien ne l'ait cité comme lieu de pélerinage ou digne de visite; l'un d'eux y fut même enterré, comme on l'a vu. La date de 1447 est également intéressante, car elle révèle que le port de Klarentsa devait toujours être fréquenté, bien que la ville eût été démantelée en 1430 par Constantin Paléologue.

Tels sont les modestes vestiges que nous avons pu réunir ${ }^{3}$; ils n'ajoutent malheureusement que fort peu à ce que nous savons de l'histoire de la principauté d'Achaie. On peut espérer que des trouvailles

1. Plusieurs de ces graffiti ont été signalés par A. K. Orlandos, 'A p . 'E $\varphi .$, 1923, p. 34, fig. $60-62$.

2. Un Francesco da Priuli est châtelain de Modon au début du XVe siècle, cf. F. Thiriet, La Romanie vénitienne, Paris 1959, pp. 198-199. Celui qui grava son nom au couvent des Vlachernes en 1447, si c'est le même que celui qui fut gouverneur de Chypre, a dû soit résider assez longtemps en Morée, soit faire un premier voyage avant celui qui le conduisit à Chypre, étant donné la différence des dates 1447-1449.

3. On pourrait ajouter, en restant hors des régions proprement vénitiennes: un blason sculpté sur une plaque encastré dans le mur Sud de l'église de la 
fortuites viendront peu à peu allonger cette liste très brève; mais, tout en tenant compte des destructions qui se sont produites dans le cours des cinq siècles qui ont suivi la fin de la principauté, on doit admettre que les Francs aux XIIIe et XIVe siècles n'avaient pas eu le même souci de marquer les monuments de leurs armes et de leurs noms que l’ont eu les Vénitiens, surtout d'ailleurs à une époque plus récente.

Antoine BON

Théotokos (Zoodochos Pigi) à Karytaina, publié par R. Tra qu a ir, A.B.S.A., XIII, 1906/07, pp. 269-271, et pl. VIII, qui l'attribue à la famille des Albamonte de Sicile, et une plaque de marbre naguère maçonnée dans le mur Nord de l'église Saint-Georges de Kavassila en Elide (l'église a été reconstruite il y a une dizaine d'années et la plaque déposée sur le sol); un écu chargé d'un arbre y était finement sculpté, au-dessus apparaissait le buste de la Vierge et, en bas, de part et d'autre, était représenté un personnage; ces deux reliefs différent nettement par le style, italien ou vénitien, des documents que nous avons étudiés, et sont sensiblement postérieurs. L'inscription de Patras publiée par A. B l o u e t, op. cit., III, p. 64 no 9, est vénitienne et postérieure au début du XVIe siècle, puisqu'elle porte die Prim(o) aprilis | md Xxxir. A. Xyngopoulos, Фparrixd

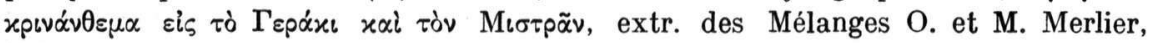
Athènes, 1953, étudiant les fleurs de lys à Geraki et à Mistra, y voit les éléments d'authentiques blasons; s'il s'agit bien de motifs qui peuvent avoir un caractère héraldique, ils n'appartiennent pas ici, je crois, à de véritables armoiries; aux Vlachernes d'Elide, à l'angle Nord-Est de l'église, sur la corniche à hauteur d'appui des fenêtre des absides, est également sculptée une fleur de lys comme motif purement décoratif. 